\title{
Ultrasound finding predictive of malignant pleural effusion
}

\author{
Brian Grondin-Beaudoin MD FRCPC, Elaine Dumoulin MD FRCPC
}

Dleural effusion is a highly common clinical presentation in malignant and benign diseases. The differential diagnosis is broad and includes heart failure, parapneumonic effusion, empyema, pulmonary emboli, inflammatory disease and malignancies, among others.

Pleural ultrasound is often used to identify the most appropriate anatomical location to perform a diagnostic or therapeutic thoracentesis. It has been shown to decrease the rate of complications associated with the procedure (1). The use of ultrasound for any pleural procedure is part of official guidelines and was given a strong recommendation in the 2010 British Thoracic Society guideline (2).
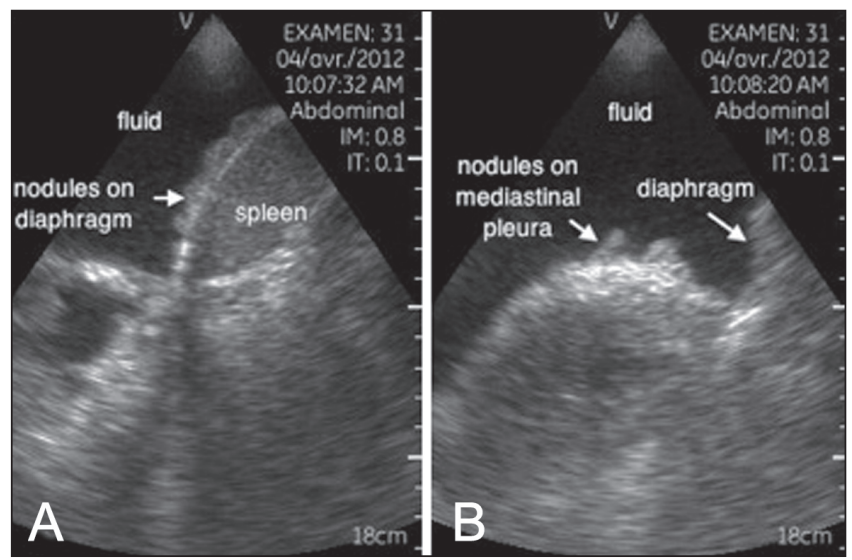

Figure 1) Ultrasound images (Vscan, GE Healthcare, USA) (1.7 MHz to $3.8 \mathrm{MHz}$ probe) of nodules on the diaphragm (A) and nodules on the mediastinal pleura (B)
Typical findings on ultrasound have been associated with malignancy. In a study involving 54 patients with pleural effusion (3), the presence of nodular thickening of the diaphragm was associated with malignancy in all cases (15 of 15). Transthoracic ultrasound correctly diagnosed malignancy in 26 of 33 patients (sensitivity 73\%, specificity $100 \%$, positive predictive value $100 \%$, negative predictive value $79 \%$ ).

A 74-year-old woman presented with a left-sided pleural effusion on chest radiography, and ultrasound images revealed the presence of nodules on the diaphragm as well as the mediastinal pleura (Figures 1 and 2A). The patient underwent thoracentesis. The cytology was positive for a metastatic adenocarcinoma of the lung to the pleura. Although a computer tomography scan (Figure 2B) was performed two weeks later, a diagnosis of stage 4 adenocarcinoma of the lung was already made.

\section{KEY LEARNING POINTS}

- Ultrasound imaging of the pleura is useful to identify the appropriate anatomical location to perform a thoracentesis, decreasing the rate of complications.

- Pleural ultrasound is also a predictive tool for malignancy. Nodules on the diaphragm or nodular thickening of the diaphragm are associated with a diagnosis of malignancy.

\section{REFERENCES}

1. Daniels CE, Ryu JH. Improving the safety of thoracentesis. Curr Opin Pulm Med 2011;4:232-6.

2. Havelock T, Teoh R, Laws D, et al. Pleural procedures and thoracic ultrasound: British Thoracic Society pleural disease guideline 2010. Thorax 2010;65(Suppl 2):ii61-76.

3. Qureshi NR, Rahman NM, Gleeson FV. Thoracic ultrasound in the diagnosis of malignant pleural effusion. Thorax 2009;65:139-43.
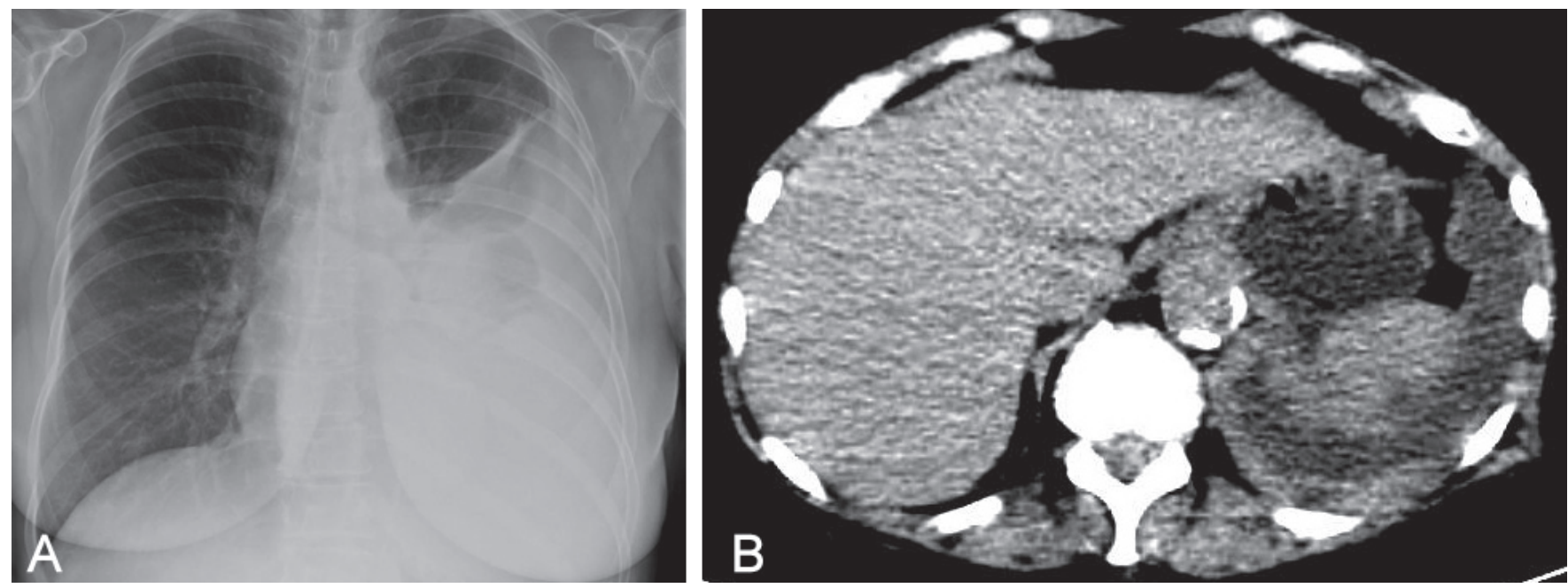

Figure 2) Left-sided pleural effusion on chest radiography (A) and computed tomography scan (B)

The 'Images in Respiratory Medicine' section of the Canadian Respiratory Journal aims to highlight the importance of visual interpretation, whether physiological, radiological, bronchoscopic, surgical/thorascopic or histological, in the diagnosis of chest diseases. Submissions should exemplify a classic, particularly dramatic or intriguing presentation of a disease while offering an important educational message to the reader (insightful diagnostic pearls or differential diagnosis, etc). This section is not intended to be a vehicle for publication of case reports (see the Clinical-Pathologic Conferences for case-based leaning series).

Centre hospitalier universitaire de Sherbrooke, Department of Respiratory Medicine, Université de Sherbrooke, Sherbrooke, Québec Correspondance: Dr Elaine Dumoulin, Département de pneumologie, Centre hospitalier universitaire de Sherbrooke, 3001, 12e Avenue Nord,

bureau 2616, Sherbrooke, Québec J1H 5N4. Telephone 819-822-6725, fax 819-822-6763, elaine.dumoulin@usherbrooke.ca 


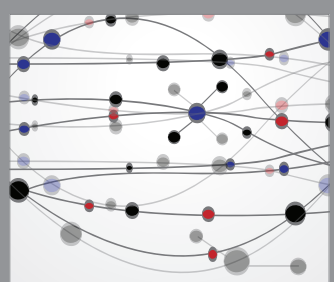

The Scientific World Journal
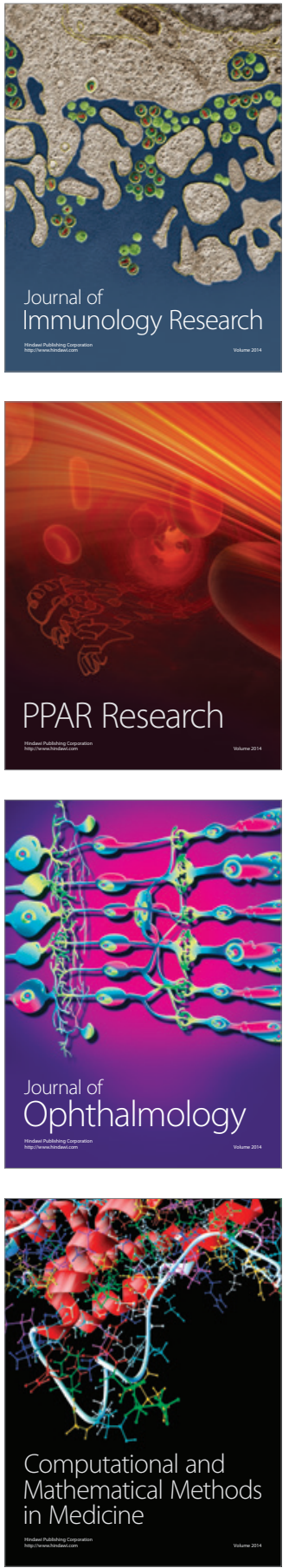

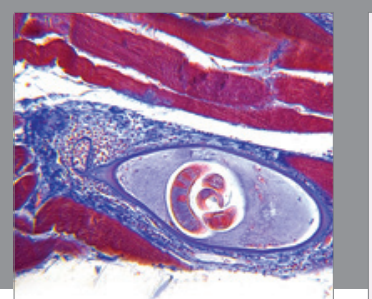

Gastroenterology Research and Practice

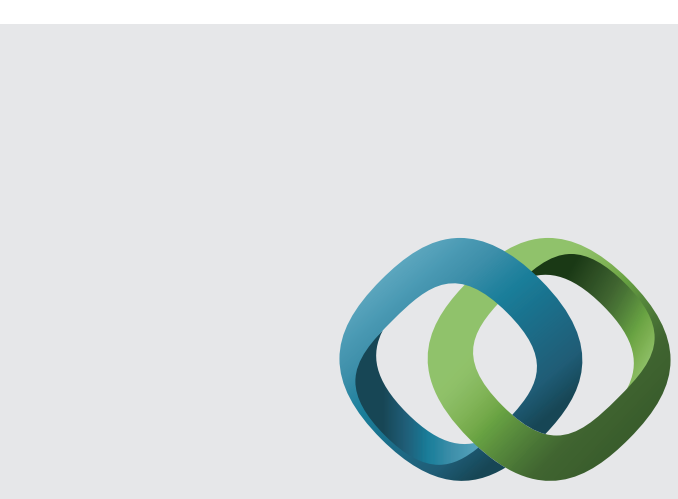

\section{Hindawi}

Submit your manuscripts at

http://www.hindawi.com
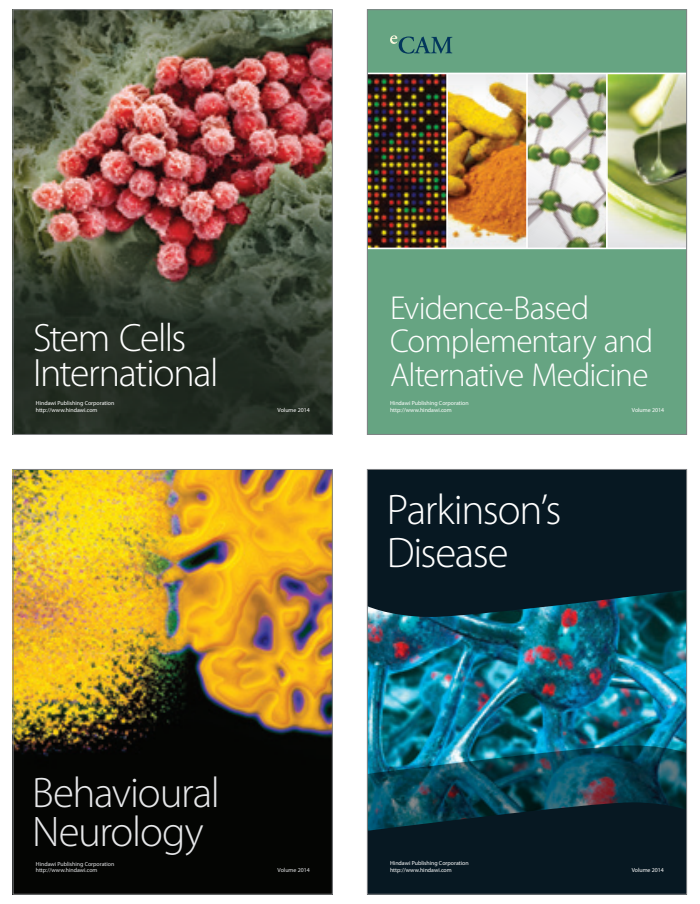
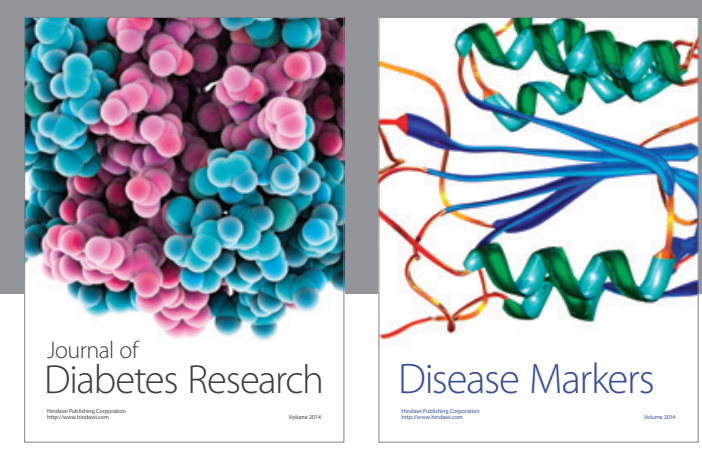

Disease Markers
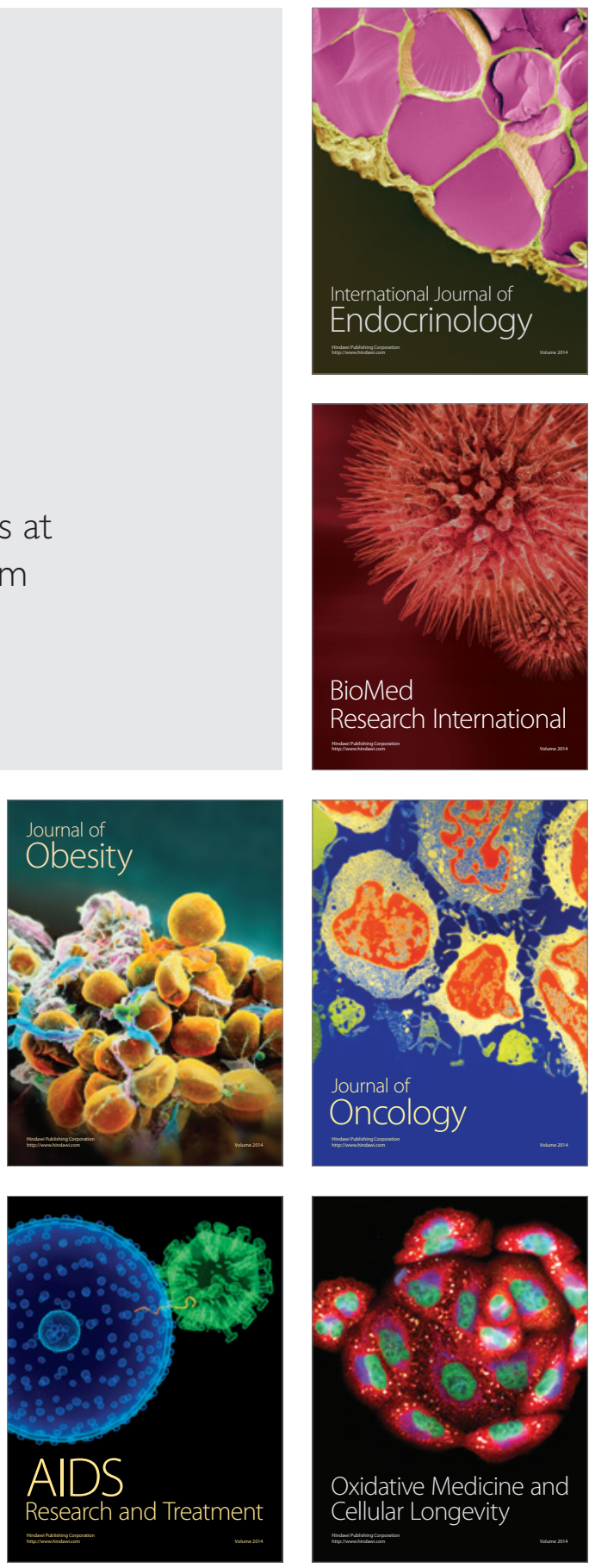